\title{
Orientation Formed by a Spot's Trajectory: A Two-Dimensional Population Approach in Primary Visual Cortex
}

\author{
Dirk Jancke \\ Institut für Neuroinformatik, Theoretische Biologie, Ruhr-Universität, D-44780 Bochum, Germany
}

There exist a large number of visual illusions indicating that perception differs from pure representation of physical input. For example, a spot of light can be characterized by its position, but it does not contribute any information about orientation. However, when moved fast enough, a continuous streak along its trajectory is perceived that helps to determine the orientation of the movement path. The question arises whether the processing of the trajectory and its orientation are simultaneously represented in the primary visual cortex. Here I show that decoding neural population activity within a twodimensional parameter space represents both (1) physical input given by the actual position of the moving spot and (2) orientation. This latter parameter has no physical counterpart in the stimulus but must be actively formed by spatiotemporal integration of the spot's trajectory.

Key words: cat; interaction; motion streak; neural ensembles; orientation preference; population code; population dynamics; receptive field; striate cortex; visual field
A fast-moving spot of light produces a continuous streak along its trajectory that can be used to extract orientation information (Geisler, 1999). Although motion streaks should hamper a clear perception of an object's actual position, there is opposite evidence that the visual system contains mechanisms to "deblur" motion smear (Burr, 1980; Castet, 1994). To solve the apparent incompatibility of both psychophysical observations, it has been hypothesized that higher visual areas are specifying the required function and thus separately compute orientation and stimulus position (Burr and Morgan, 1997). In any case, the questions remain regarding how a moving spot can form orientation and how positional and orientation encoding is segregated along the visual pathway.

Dependent on context, a large number of visual illusions [e.g., the perception of illusory contours (Kanizsa, 1976; von der Heydt et al., 1984; Grosof et al., 1993; Ramachandran et al., 1994; Sheth et al., 1996; Anderson et al., 1999; Mendola et al., 1999)] indicate that the visual system must contain mechanisms leading to representational spaces that have no physical counterpart in the stimulus. Probably the best-known neural code that represents a "visual" illusion per se is used in trichromatic color vision (Young, 1802). The joint activation of a population of retinal receptors tuned to different wavelengths can lead to the sensation of "white," which is not defined in the input space of any single wavelength (Lehky and Sejnowski, 1999). Experimentally the problem arises of (1) how to relate neural activity to several parameter values simultaneously and (2) how to extract the representation of parameters that are not explicitly defined by the input.

\footnotetext{
Received Jan. 18, 2000; revised May 11, 2000; accepted May 12, 2000.

This work was supported by the Deutsche Forschungsgemeinschaft, Schwerpunkt "Physiologie und Theorie neuronaler Netzwerke." This work would not have been possible without the framework contributed by Dr. Gregor Schöner and Dr. Hubert Dinse. I thank Dr. Axel Steinhage and Dori Derdikman for comments on the Materials and Methods section and Dr. Steinhage also for implementation of the OLE in MATLAB. I thank Drs. Werner von Seelen, Klaus-Peter Hoffmann, Wilson S. Geisler, Frederic Chavane, Eyal Seidemann, and Amos Arieli for helpful discussion. I am grateful to Dr. Christoph Schreiner for constructive proofreading of an earlier version of this manuscript. I thank M. Neef and M. Ziesmer and the members of the mechanical shop for excellent technical support.

Correspondence should be addressed to Dr. Dirk Jancke, Research of Higher Brain Functions, Weizmann Institute of Science, P.O. Box 26, 76100 Rehovot, Israel. E-mail: dirk.jancke@weizmann.ac.il.

Copyright (C) 2000 Society for Neuroscience $\quad 0270-6474 / 00 / 200001-06 \$ 15.00 / 0$
}

We have recently introduced a population coding technique that allows for investigation of cooperative processes in cat area 17 (Jancke et al., 1996, 1999). Our studies demonstrated systematic deviations of the population representations from a simple retinotopic projection of the visual input. Such differences were interpreted as signatures of neural interaction dependent on stimulus context. Generally, neural population analysis refers to the notion that ensembles of neurons, each coarsely tuned to different but overlapping ranges of parameter values, contribute to a common representation of sensory or motor parameters (Georgopoulos et al., 1986; Steinmetz et al., 1987; Gielen et al., 1988; Lee et al., 1988; Vogels, 1990; Young and Yamane, 1992; Zohary, 1992; Seung and Sompolinsky, 1993; Wilson and McNaughton, 1993; Nicolelis and Chapin, 1994; Sugihara et al., 1998; Pouget et al., 1998; Zemel et al., 1998; Zhang et al., 1998; Deneve et al., 1999; Erlhagen et al., 1999; Stanley et al., 1999).

In the present study, the population approach has been extended to a simultaneous analysis of two parameters: visual field position and orientation. Traditionally, orientation tuning has been investigated presenting bar-shaped stimuli (Hubel and Wiesel, 1962) or drifting gratings (Campbell et al., 1968). Here, the cortical representation of orientation was analyzed by presenting horizontally moved spots of light within the central visual field of cat area 17. The results suggest that the neural population recovers the orientation of the moving spot's trajectory by spatiotemporal integration and segregates the positional information from the orientation signal sequentially in time.

This article is published in The Journal of Neuroscience, Rapid Communications Section, which publishes brief, peer-reviewed papers online, not in print. Rapid Communications are posted online approximately one month earlier than they would appear if printed. They are listed in the Table of Contents of the next open issue of JNeurosci. Cite this article as: JNeurosci, 2000, 20:RC86 (1-6). The publication date is the date of posting online at www.jneurosci.org.

http://www.jneurosci.org/cgi/content/full/4377 


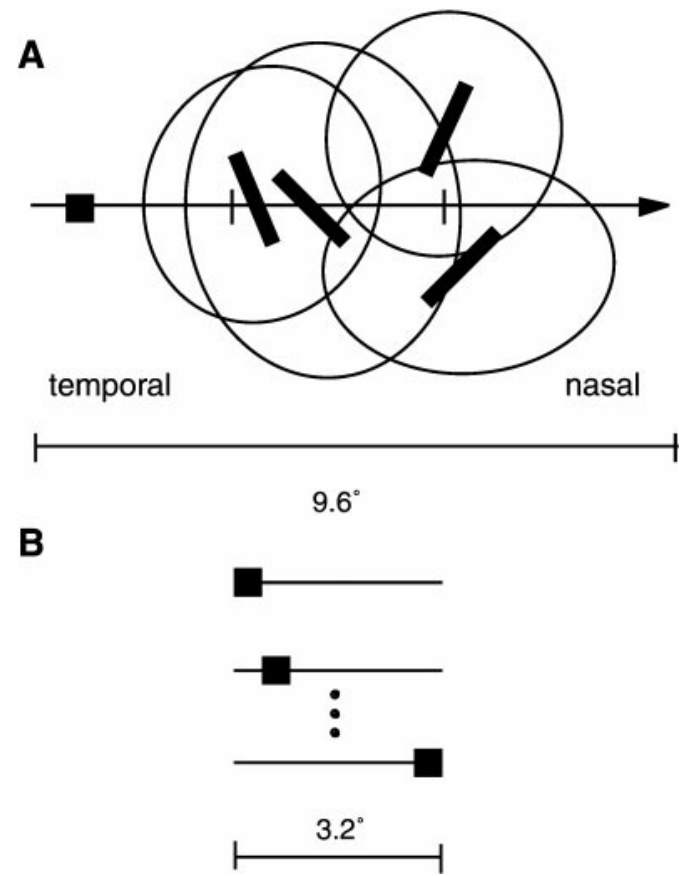

Figure 1. Schematic illustration of the stimulus configurations. $A$, Small squares of light $\left(0.4 \times 0.4^{\circ}\right)$ were moved horizontally along the central visual field representation of cat area 17 at four different speeds $(4.5,8.8$, $\left.15.1,38.4^{\circ} / \mathrm{sec}\right)$. The central part of the stimulus trajectory $\left(3.2^{\circ}\right.$, indicated by small vertical lines) was analyzed by recording RFs that densely overlapped within this visual field portion (as indicated by ellipsoids). The length of the entire trajectory was $9.6^{\circ}$. For optimal linear estimation of orientation, a bar-shaped stimulus $\left(0.4 * 3.2^{\circ}\right)$ was flashed $(25 \mathrm{msec})$ at eight different angles at the RF center of each neuron (exemplified by oriented black bars). $B$, For estimation of position, squares were flashed for $25 \mathrm{msec}$ at eight contiguous locations within the sampled visual space.

\section{MATERIALS AND METHODS}

Animals and preparation. Extracellular recordings from a total of 178 cells were made in the central visual field representation of the left hemisphere of area 17. Twenty adult cats of both sexes were used. Treatment of all animals was within the regulations of the National Institution of Health Guide and Care for Use of Laboratory Animals (Rev. 1987). During surgery and recording, anesthesia was maintained by artificial respiration with a mixture of $75 \% \mathrm{~N}_{2} \mathrm{O}$ and $25 \% \mathrm{O}_{2}$ and by application of sodium pentobarbital (Nembutal, $3 \mathrm{mg} \cdot \mathrm{kg}^{-1} \cdot \mathrm{hr}^{-1}$, i.v.; Ceva). Animals were paralyzed by continuous infusions of gallamine triethiodide $\left(2 \mathrm{mg} / \mathrm{kg}\right.$, i.v. bolus, $2 \mathrm{mg} \cdot \mathrm{kg}^{-1} \cdot \mathrm{hr}^{-1}$, i.v.; Sigma, St. Louis, $\mathrm{MO}$ ). Heart rate, intratracheal pressure, expired $\mathrm{CO}_{2}$, body temperature, and EEG were monitored during the entire experiment. Contact lenses with artificial pupils ( $3 \mathrm{~mm}$ diameter) were used to cover the eyes, which were frequently rinsed with artificial eye liquid (for details see Jancke et al., 1999).

Recording and stimulation. Recordings were performed with two glasscoated platinum electrodes (resistance between 3.5 and 4.5 MOhm; Thomas-Recording). Electrode signals were fed into spike sorters based on an on-line principal component analysis (T. J. Gawne and B. J. Richmond, National Institutes of Health, Bethesda, MD). As a rule, two cells were recorded simultaneously. Stimuli were displayed on a PCcontrolled 21 inch monitor $(120 \mathrm{~Hz}$, noninterlaced) positioned at a distance of $114 \mathrm{~cm}$ from the animal. Luminance of the stimuli was 0.9 $\mathrm{cd} / \mathrm{m}^{2}$, and background luminance was $0.002 \mathrm{~cd} / \mathrm{m}^{2}$. Smooth stimulus trajectories (temporonasal) were generated by varying the stimulus shift per video frame $(8.3 \mathrm{msec})$ resulting in different speeds $(4.5,8.8,15.1$, and $38.4^{\circ} / \mathrm{sec}$ ). The length of the entire stimulus trajectory was $9.6^{\circ}$. Analysis was restricted to the central $3.2^{\circ}$ of the trajectory as indicated by small vertical lines in Figure $1 A$. Stimuli were presented in pseudorandom order to the contralateral eye (32 stimulus repetitions). The retinal position of the stimuli was constant, regardless of the receptive field (RF) location of the individual neurons (non-RF-centered, but stimuluscentered approach illustrated in Fig. 1A). To control for eye drift, RF locations were measured repeatedly.
Construction of population representations. For the construction of population representations across position and orientation, an optimal linear estimator (OLE) was used. Originally, this technique was introduced to reconstruct a single value of an encoded quantity from the firing rates of an ensemble of neurons (Salinas and Abbott, 1994). Here, this technique was extended to directly estimate entire distributions of population activity defined in the visual field and in the orientation space (cf. Zemel et al., 1998; Pouget et al., 1998; Jancke et al., 1999, for similar approaches).

Spiking activity was recorded from 178 neurons with RFs that densely overlapped within the sampled visual space. Two identical sets of stimuli were presented to each neuron. (1) To sample the visual field along the central part of $3.2^{\circ}$ of stimulus trajectories, eight single squares of $0.4^{\circ}$ were flashed $(25 \mathrm{msec})$ at adjacent positions (see Fig. 1B). (2) For sampling orientation, bars $\left(0.4^{*} 3.2^{\circ}\right)$ were flashed $(25 \mathrm{msec})$ at eight different angles $\left(0-157.5^{\circ}\right)$ within the RF center of each neuron (see Fig. $1 A$, bars; the center of the RF of each cell was computed as the centroid of the smoothed RF profile) (Jancke et al., 1999).

(1) The estimation for visual field position is based on the responses to eight flashed squares of light. $\hat{U}_{\mathrm{i}}\left(s_{\mathrm{k}}\right)$ is the distribution of population activity (population representation) for each square at the position $s_{\mathrm{i}}$. The number $M$ of sample points $s_{\mathrm{k}}$ determines the degree of resolution with which the activity distributions are calculated. Each neuron $(n=1$, $\ldots, 178)$ contributes to the entire population with a set of coefficients, $c_{\mathrm{n}}\left(s_{\mathrm{k}}\right)$, to be determined by optimization, and its firing rates, $f_{\mathrm{n}}\left(s_{\mathrm{i}}\right)$, in response to each square, $s_{i}$. The firing rates were averaged over the time interval between 40 and $65 \mathrm{msec}$ after stimulus onset corresponding to the peak responses in the poststimulus time histograms (the exact size of the integration window is not critical for the estimation procedure):

$$
\hat{U}_{\mathrm{i}}\left(s_{\mathrm{k}}\right)=\sum_{\mathrm{n}=1}^{\mathrm{N}} c_{\mathrm{n}}\left(s_{\mathrm{k}}\right) f_{\mathrm{n}}\left(s_{\mathrm{i}}\right) .
$$

A Gaussian was chosen as the desired shape of $U_{\mathrm{i}}\left(s_{\mathrm{k}}\right)$, centered around each of the eight stimulus positions, $s_{\mathrm{i}}$ :

$$
U_{\mathrm{i}}\left(s_{\mathrm{k}}\right)=\exp \left(-\frac{\left(s_{\mathrm{k}}-s_{\mathrm{i}}\right)^{2}}{2 \sigma^{2}}\right) \text { with } s_{\mathrm{k}} \in\left[s_{1}-\sigma, s_{8}+\sigma\right], k=1 \ldots M .
$$

The shape of the Gaussian ( $\sigma=0.6^{\circ}$ in visual space) approximately matched the average RF profile of all neurons measured (Jancke et al., 1999). To determine the coefficients, $c_{\mathrm{n}}\left(s_{\mathrm{k}}\right)$, the average reconstruction error $\Sigma_{\mathrm{i}}\left(\hat{U}_{\mathrm{i}}\left(s_{\mathrm{k}}\right)-U_{\mathrm{i}}\left(s_{\mathrm{k}}\right)\right)^{2}$ was minimized (Salinas and Abbott, 1994; Pouget et al., 1998), which leads to:

$$
c_{\mathrm{n}}\left(s_{\mathrm{k}}\right)=\sum_{\mathrm{m}=1}^{\mathrm{N}} L_{\mathrm{m}}\left(s_{\mathrm{k}}\right) Q_{\mathrm{nm}}^{-1} .
$$

Here, $Q_{\mathrm{nm}}$ is the correlation matrix between the firing rates of neurons $n$ and $m$ for all stimuli:

$$
Q_{\mathrm{nm}}=\sum_{\mathrm{i}=1}^{8} f_{\mathrm{n}}\left(s_{\mathrm{i}}\right) f_{\mathrm{m}}\left(s_{\mathrm{i}}\right),
$$

and $L_{\mathrm{m}}\left(s_{\mathrm{k}}\right)$ is:

$$
L_{\mathrm{m}}\left(s_{\mathrm{k}}\right)=\sum_{\mathrm{i}=1}^{8} U_{\mathrm{i}}\left(s_{\mathrm{k}}\right) f_{\mathrm{m}}\left(s_{\mathrm{i}}\right) .
$$

(2) The analogous procedure was performed for eight angles of flashed bar stimuli, $s_{\varphi}$. As the desired shape of the population distributions, Gaussians (width, $\sigma=33.75^{\circ}$, calculated with $M$ sample points $s_{\phi}$ ) were chosen that approximately fitted the shape of the orientation tuning curve of the typical area 17 cell (for review, see Orban, 1984). Also cosines were tested showing that the results are not critically dependent on the exact shape of the distributions.

The crucial step consists of extrapolating the neural responses recorded for moving stimuli onto the predefined two-dimensional parameter space. The estimators were used to obtain time-resolved population representations by replacing the firing rate $f_{\mathrm{n}}\left(s_{\mathrm{i}}\right)$ in Equation 1 with the 


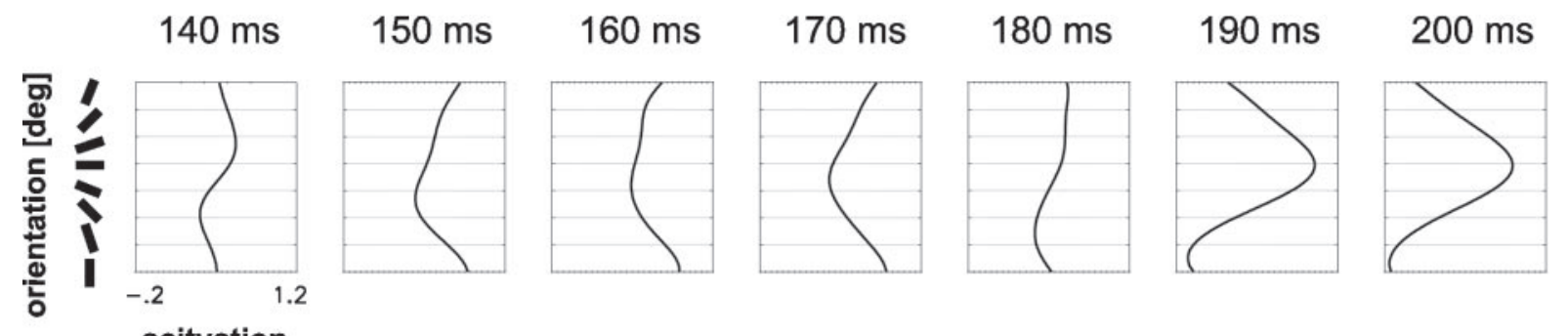

acitvation
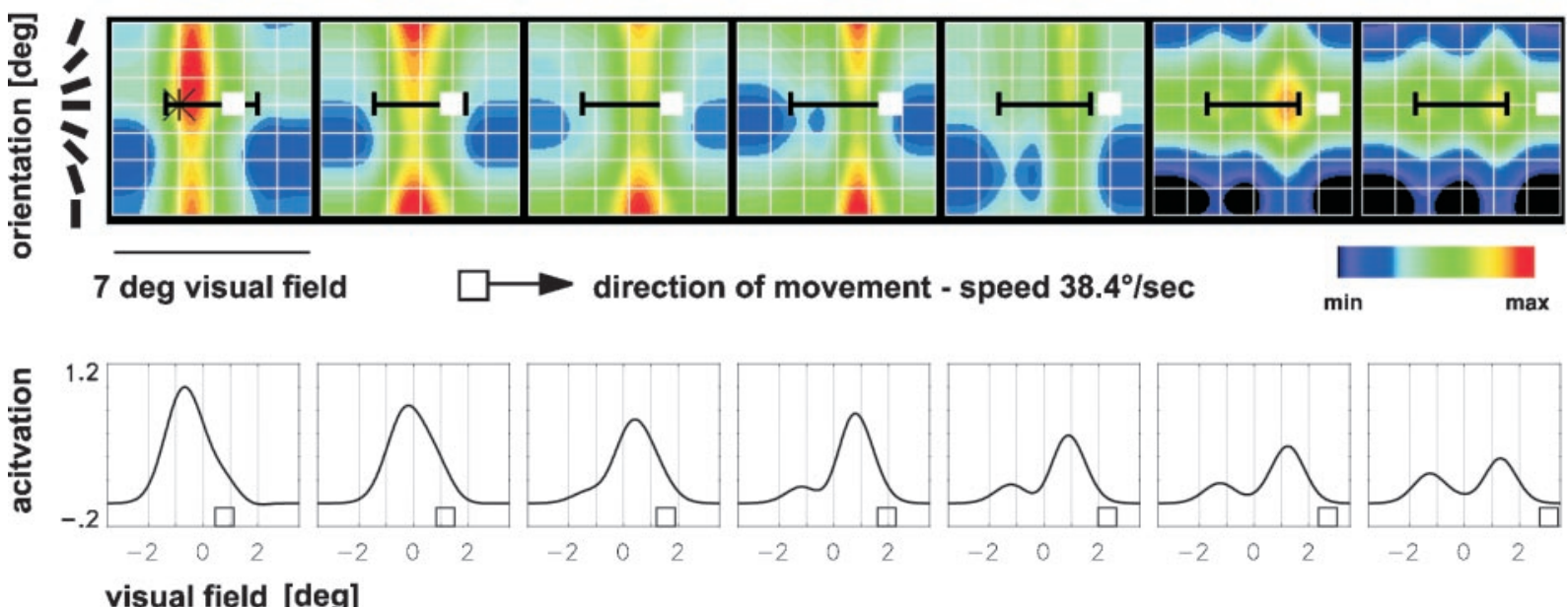

Figure 2. Population representation of a fast moving spot. Top panel, Distribution of activity within orientation space (values for orientation are indicated by bars). Depicted are sequences of activity distributions resolved at time steps of 10 msec running from left to right starting 140 msec after stimulus onset. Middle panel, Two-dimensional population representation of visual space ( $x$-axis) and orientation ( $y$-axis) by summing across orientation (top panel) and visual space (bottom panel). White square marks the actual stimulus position; color scale indicates level of response strength. Starting point of the stimulus was $3.2^{\circ}$ outside the sampled visual space outlined in black. Bottom panel, Distribution of population activity in visual space. The actual position of the moving stimulus is indicated at the bottom of each picture. The population representations were normalized to maximum activity over the entire time of responses.

firing rate for moving stimuli, $f_{\mathrm{n}}(m(t))$, in a particular time interval. The coefficients $c_{\mathrm{n}}\left(s_{\mathrm{k}}\right)$ and $\tilde{c}_{\mathrm{n}}\left(s_{\phi}\right)$, by contrast, remained fixed:

$$
\begin{gathered}
\hat{U}^{\mathrm{mo}-\mathrm{pos}}\left(s_{\mathrm{k}}, t\right)=\sum_{\mathrm{n}=1}^{\mathrm{N}} c_{\mathrm{n}}\left(s_{\mathrm{k}}\right) f_{\mathrm{n}}(m(t)) \\
\hat{U}^{\mathrm{mo}-\text { ori }}\left(s_{\Phi}, t\right)=\sum_{\mathrm{n}=1}^{\mathrm{N}} \tilde{c}_{\mathrm{n}}\left(s_{\Phi}\right) f_{\mathrm{n}}(m(t)) .
\end{gathered}
$$

$\hat{U}^{\text {mo-pos }}\left(s_{\mathrm{k}}, t\right)$ and $\hat{U}^{\text {mo-ori }}\left(s_{\phi}, t\right)$ are distributions of population activity based on firing rates that are observed in response to spots of light moving at different velocities. In general, the construction of predefined representational spaces incorporates the option to treat neural responses with respect to parameters that have not explicitly been chosen as an actual physical input, such as orientation in case of a spot's trajectory. $\hat{U}^{\text {mo-ori }}\left(s_{\phi}, t\right)$, coding for orientation (see Fig. 2, top panel) and $\hat{U}^{\text {mo-pos}}\left(s_{\mathrm{k}}, t\right)$, coding for visual field position (see Fig. 2, bottom panel) were summed point by point across both dimensions. As a result, population representations within a two-dimensional position-orientation space were obtained (see Fig. 2, middle panel). Multiplication of the distributions yielded almost identical results showing that the linear assumption here is not critical. Because the activity distributions were summed at the population level, they roughly reflect an average across all positions and orientations. Therefore, they are subject to a linearizing effect similar to that reported previously for patterns of population activity (Arieli et al., 1996). However, for the distribution of neurons in a single recording session a more prominent nonlinear behavior could still be expected.

\section{RESULTS}

\section{Dynamics of population activity within a position-orientation space}

A two-dimensional parameter space was predefined using a set of stimuli that consisted of squares flashed at contiguous positions and bars flashed at different angles (Fig. 1A,B). Homogeneous distributions of population activity were calculated assuming that neurons are commonly contributing to Gaussian-shaped representations (distributions of population activity) of these stimuli. To construct activity distributions, an optimal linear estimator was used that optimizes the normalization of the cell responses under the condition of a well defined least squares fit. Thereby, the optimal estimator takes into consideration the relationship between neurons within each parameter space and takes small irregularities of sampling density into account (see Materials and Methods).

The aim of the study was to investigate how a moving spot is represented within the predefined two-dimensional positionorientation space. Moving stimuli are likely to cause strong modulations of the neural firing rates indicating important interactions in response to a dynamic visual context (Tolhurst and Heeger, 1997). A small spot of light of $0.4^{\circ}$ width was moved temporonasally at $38.4^{\circ} \mathrm{sec}$. Figure 2 shows the evolution of population activity depicted in $10 \mathrm{msec}$ time slices. The time indicated on top was always related to motion onset, which was $3.2^{\circ}$ outside the sampled fraction of the visual field. The middle panel demonstrates the population activity in the twodimensional space with position on the $x$-axis and orientation on the $y$-axis. Because of neural delay times, cell responses could be observed after the stimulus (white square) has passed a certain portion of the sampled space. The time required for the entire stimulus passage was $250 \mathrm{msec}$. The population pattern within this two-dimensional parameter space is characterized by a narrow band of activity that kept on propagating along the stimulus trajectory indicative for a spatially and temporally precise coding of position (140-180 msec). Throughout this time period the 
A

subsequent representation

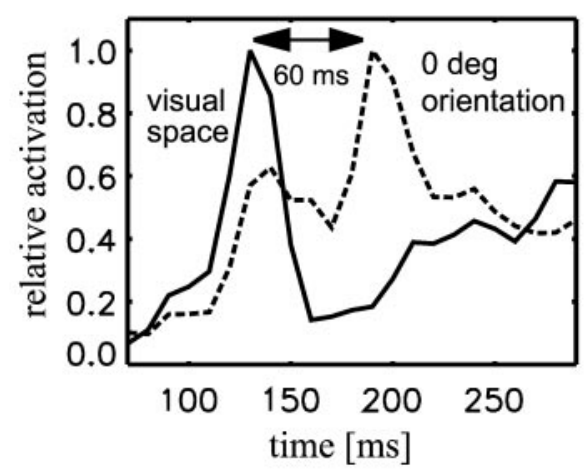

B

sharpness of orientation

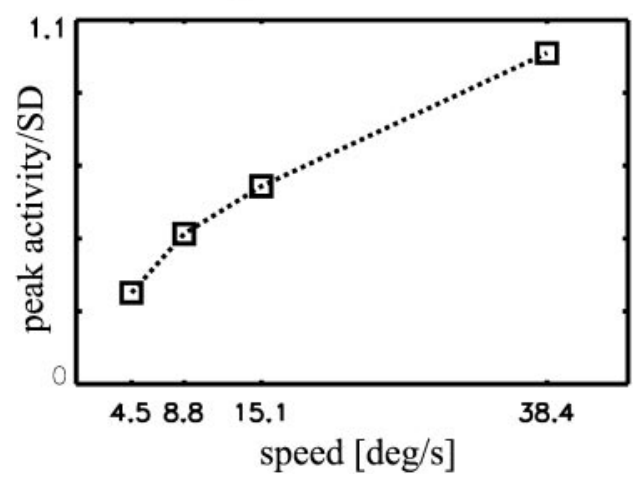

Figure 3. A, Evolution of activity depicted for visual space (solid line) and for orientation (stippled line) as a function of time after stimulus onset. Activity was read out at one particular location within each dimension (marked by asterisk in Fig. 2, middle panel, left time window). Amplitudes were normalized to maximal activity for each parameter separately. $B$, Sharpness of population representation for orientation dependent on stimulus speed. Sharpness was calculated by dividing maximal peak amplitudes by the SDs.

representation of position was basically unrelated to orientation preference as can be inferred from the vertical pattern of activation along the orientation axis. During the time interval between 150 and $170 \mathrm{msec}$, activity showed some selectivity for orientations perpendicular to movement axis (in accordance with Henry et al., 1974) because the maximum activity was preferentially located around 90 and $157.5^{\circ}$ (vertical) orientation.

This behavior changed when the stimulus had passed a larger portion of its trajectory. At that time, activity became specific for horizontal orientation resulting in a narrow horizontal band of activity along the position axis. In terms of positional coding, activity became smeared out in space (cf. time steps around 190 msec). Figure 2, bottom panel, shows the population activity for the parameter position separately. A coherently propagating distribution of activity can be observed following the moving stimulus (shown at bottom line) at its respective speed. In the later part of the response the distribution became flat and slightly bimodal, whereas the opposite behavior can be seen in the orientation space (Fig. 2, top panel). In this epoch, the peak of population activity is representing horizontal $\left(0^{\circ}\right)$ orientation.

Figure $3 A$ shows the evolution of activity read out at one particular location of the population representations (Fig. 2, asterisk, middle panel, left time window). Activity representing visual position reached its maximal level $60 \mathrm{msec}$ before the maximal amplitude representing horizontal orientation. This demon- strates that the population subsequently represents both the shifting stimulus and horizontal orientation formed by spatiotemporal integration of the moving spot's trajectory.

\section{Forming of orientation depends on motion speed}

To calculate the sharpness of orientation representation, I divided the peak amplitudes of the activity distributions by the SDs (Fig. $3 B$ ). The sharpness of the motion-induced orientation tuning depends on the speed of the stimulus. This observation is not unexpected assuming that motion smear (1) is a prerequisite for generating information about orientation and (2) is largely dependent on higher speeds attributable to the temporal integration properties of the visual system (Burr, 1980). In a recent psychophysical study, the representation of orientation was tested by presenting a moving dot together with a parallel oriented line mask. In that study, human thresholds for the discrimination of the orientation of the dot's trajectory were improved with higher speeds (Geisler, 1999).

\section{How many neurons are needed to give a reliable population response?}

To rule out the possibility that the results were biased by cell number or sampling, a bootstrap method was applied (Zoubir and Boashash, 1998). In this procedure, neurons were randomly selected from the pool of the entire population (iterated 20 -fold per number of cells). Figure 4 shows population representations of orientation as a function of the number of neurons for a stimulus speed of $38.4^{\circ} / \mathrm{sec}$. The formation of orientation preference along the horizontal stimulus trajectory seems to be nearly independent of the number of cells when a minimum requirement of $\sim 80$ neurons was exceeded (Fig. $4 B, C$ ). This suggests that the results are only minimally affected by the recording parameters of cell number and sampling uniformity.

\section{DISCUSSION}

A challenging issue in experimental neurobiology is to investigate how the visual system actively creates features that are not present in the light patterns striking the retina. The data obtained here provide evidence that area 17 can form orientation by spatiotemporal integration of a spot's trajectory. Two features were subsequently computed. Whereas the first part of the response accounts for a sharp representation of the stimulus in visual space, the later part revealed uncertainty in localization ("motion smear") and codes for horizontal orientation. Such a processing strategy may be used to dynamically convey information to higher visual areas.

\section{Analyzing neural populations}

Most of the neurons were not stimulated within their RF centers. In everyday life, visual objects are similarly distributed in arbitrary ways across RFs, so that this way of stimulus presentation and averaging is crucial for an understanding of how complex scenes are represented in visual cortex. It is well established that widespread patterns of cortical activation are evoked while even very small visual objects are processed (cf. Grinvald et al., 1994). These patterns are believed to reflect the complex organization of the visual cortex leading to activation of the mass activity of groups of neurons that simultaneously process a diversity of feature characteristics (Hubel and Wiesel, 1962; Orban, 1984; Livingstone and Hubel, 1988). Increasing evidence indicates that neural activity in area 17 depends on context and strongly varies with more complex visual features reflecting the functional state of an extended cortical network (Das and Gilbert, 1999; Eysel, 1999; Tsodyks et al., 1999). Specifically, the accurate perception of object location during motion involves a fine spatiotemporal interplay between excitatory and inhibitory processing among widely connected and interacting neural populations (Gegenfurt- 

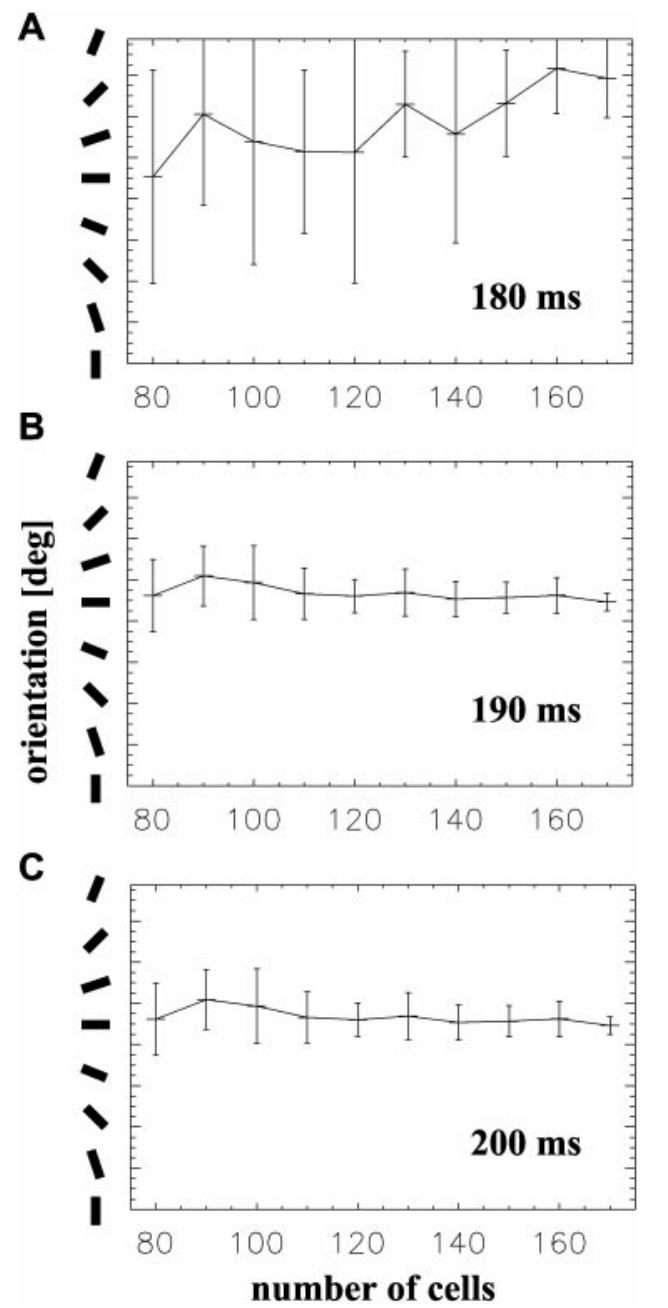

Figure 4. Bootstrap analysis. Representation of orientation within a neural population induced by a moving spot of light. Shown are three 10 msec time windows; time after stimulus onset is indicated. Different populations including different numbers of cells were built by randomly selecting (20 iterations) from a pool of 178 neurons (vertical bars show SDs). Orientation preference was calculated by reading out the maxima of the representations at each time window. $A, 180 \mathrm{msec}$ after stimulus onset there is a still a large scatter within the population representation in orientation space, indicated by the large amount of SDs. In contrast, from $190 \mathrm{msec}$ after stimulus onset $(B-C)$, the sharp representation of horizontal orientation $\left(0^{\circ}\right)$ is nearly independent of the actual sampling.

ner and Hawken, 1996) through long-range horizontal connections (Fisken et al., 1975; Gilbert, 1992; Bringuier et al., 1999).

In view of the present study, two main reasons may suggest simultaneous recording from large neural populations in future experiments. First, the population responses were obtained by averaging a limited number of cells from different animals. Synchronous recording might reduce the inherent variability of sequentially measured neurons. Second, the population representations are depicted in physical metrics. Figure 2, middle panel, intuitively implies the question of how the observed activation patterns might be implemented within the cortical anatomy. For instance, some parameters such as retinal location or selectivity for orientation seem to be systematically mapped on the cortex. However, there are remarkable distortions of their topographic organization (Bonhoeffer and Grinvald, 1993; Das and Gilbert, 1997). A reasonable explanation for this is that the twodimensional cortical architecture must deal with a highdimensional input space.

\section{High-dimensional parameter spaces and cortical coordinates}

Estimating population representations of particular parameters is a method for constructing subspaces of the potentially highdimensional space of visual stimulus features. In this study, each neuron could be thought of as a point in a two-dimensional parameter space with its activity simultaneously contributing to the representation of stimulus position and orientation. The construction of multidimensional distributions of population activity that are defined in physical metrics can help to find underlying neural transformation strategies that map visual stimulus parameters onto cortical coordinates. The current study supports this concept and reveals specific aspects of the dynamics of cortical network interactions.

\section{REFERENCES}

Anderson BL, Barth HC (1999) Motion-based mechanisms of illusory contour synthesis. Neuron 24:433-441.

Arieli A, Sterkin A, Grinvald A, Aertsen A (1996) Dynamics of ongoing activity: explanation of the large variability in evoked cortical responses. Science 273:1868-1871.

Bonhoeffer T, Grinvald A (1993) The layout of iso-orientation domains in area 18 of cat visual cortex: optical imaging reveals a pinwheel-like organization. J Neurosci 13:4157-4180.

Bringuier V, Chavane F, Glaeser L, Fregnac Y (1999) Horizontal propagation of visual activity in the synaptic integration field of area 17 neurons. Science 283:695-699.

Burr D (1980) Motion smear. Nature 284:164-165.

Burr DC, Morgan MJ (1997) Motion deblurring in human vision. Proc R Soc Lond B Biol Sci 264:431-436.

Campbell FW, Cleland BG, Cooper GF, Enroth-Cugell C (1968) The angular selectivity of visual cortical cells to moving gratings. J Physiol (Lond) 198:237-250.

Castet E (1994) Effect of the ISI on the visible persistence of a stimulus in apparent motion. Vision Res 34:2103-2114.

Das A, Gilbert CD (1997) Distortions of visuotopic map match orientation singularities in primary visual cortex. Nature 387:594-598.

Das A, Gilbert CD (1999) Topography of contextual modulations mediated by short-range interactions in primary visual cortex. Nature 399:655-661.

Deneve S, Latham PE, Pouget A (1999) Reading population codes: a neural implementation of ideal observers. Nat Neurosci 2:740-745.

Erlhagen W, Bastian A, Jancke D, Riehle A, Schöner G (1999) The distribution of neuronal population activation (DPA) as a tool to study interaction and integration in cortical representations. J Neurosci Methods 94:53-66.

Eysel U (1999) Turning a corner in vision research. Nature 399:641-644.

Fisken RA, Garey CJ, Powell TPS (1975) The intrinsic, association and commissural connections of area 17 of the visual cortex. Trans R Soc Lond B Biol Sci 272:487-536.

Gegenfurtner KR, Hawken MJ (1996) Interaction of motion and color in the visual pathways. Trends Neurosci 19:394-400.

Geisler WS (1999) Motion streaks provide a spatial code for motion. Nature 400:65-69.

Georgopoulos AP, Schwartz AB, Kettner RE (1986) Neural population coding of movement direction. Science 233:1416-1419.

Gielen CCAM, Hesselmans GHFM, Johannesma PIM (1988) Sensory interpretation of neural activity patterns. Math Biosci 88:14-35.

Gilbert CD (1992) Horizontal integration and cortical dynamics. Neuron 9:1-13.

Grinvald A, Lieke E, Frostig RD, Hildesheim R (1994) Cortical point spread function and long range lateral interactions revealed by real time optical imaging of macaque monkey primary visual cortex. J Neurosci 14:2545-2568.

Grosof DH, Shapley RM, Hawken MJ (1993) Macaque V1 neurons can signal "illusory" contours. Nature 365:550-552.

Henry GH, Bishop PO, Dreher B (1974) Orientation, axis and direction as stimulus parameters for striate cells. Vision Res 14:767-777.

Hubel DH, Wiesel TN (1962) Receptive fields, binocular interactions and functional architecture in the cat's visual cortex. J Physiol (Lond) 160:559-568.

Jancke D, Akhavan AC, Erlhagen W, Schöner G, Dinse HR (1996) Reconstruction of motion trajectories from the dynamic population representation of neurons in cat visual cortex. Soc Neurosci Abstr 22:646.

Jancke D, Erlhagen W, Dinse HR, Akhavan AC, Giese M, Steinhage A, Schöner G (1999) Parametric population representation of retinal location: neuronal interaction dynamics in cat primary visual cortex. J Neurosci 19:9016-9028. 
Lee C, Rohrer WH, Sparks DL (1988) Population coding of saccadic eye movements by neurons in the superior colliculus. Nature 332:357-360.

Lehky SR, Sejnowski TJ (1999) Seeing white: Qualia in the context of decoding population codes. Neural Comput 11:1261-1280.

Livingstone MS, Hubel DH (1988) Segregation of form, color, movement, and depth: anatomy, physiology, and perception. Science 240:740-749.

Kanizsa G (1976) Subjective contours. Sci Am 234:48-52.

Orban GA (1984) Neuronal operations in the visual cortex. In: Studies of brain function XI (Braitenberg V, ed), pp 149-161. New York: Springer.

Mendola JD, Dale AM, Fischl B, Liu AK, Tootell BH (1999) The representation of illusory and real contours in human cortical visual areas revealed by functional magnetic resonance imaging. J Neurosci 19:8560-8572.

Nicolelis MAL, Chapin JK (1994) Spatiotemporal structure of somatosensory responses of many-neuron ensembles in the rat ventral posterior medial nucleus of the thalamus. J Neurosci 14:3511-3532.

Pouget A, Zhang K, Latham PE (1998) Statistically efficient estimation using population coding. Neural Comput 10:373-401.

Ramachandran VS, Ruskin D, Cobb S, Rogers-Ramachandran D (1994) On the perception of illusory contours. Vision Res 34:3145-3152.

Salinas E, Abbott LF (1994) Vector reconstruction from firing rates. J Comp Neurosci 1:89-107.

Seung HS, Sompolinsky H (1993) Simple models for reading neuronal population codes. Proc Natl Acad Sci USA 90:10749-10753.

Sheth BR, Sharma J, Rao SC, Sur M (1996) Orientation maps of subjective contours in visual cortex. Science 274:2110-2115.

Stanley GB, Li FF, Dan Y (1999) Reconstruction of natural scenes from ensemble responses in the lateral geniculate nucleus. $\mathrm{J}$ Neurosci 19:8036-8042

Steinmetz MA, Motter BC, Duffy CJ, Mountcastle VB (1987) Func- tional properties of parietal visual neurons: radial organization of directionalities within the visual field. J Neurosci 7:177-191.

Sugihara T, Edelman S, Tanaka K (1998) Representation of objective similarity among three-dimensional shapes in the monkey. Biol Cybern 78:1-7.

Tolhurst DJ, Heeger DJ (1997) Comparison of contrast-normalization and threshold models of the responses of simple cells in cat striate cortex. Vis Neurosci 14:293-309.

Tsodyks M, Kenet T, Grinvald A, Arieli A (1999) Linking spontaneous activity of single cortical neurons and the underlying functional architecture. Science 286:1943-1946.

Vogels R (1990) Population coding of stimulus orientation by striate cortical cells. Biol Cybern 64:24-31.

von der Heydt R, Peterhans E, Baumgartner G (1984) Illusory contours and cortical neuron responses. Science 224:1260-1262.

Wilson MA, McNaughton B (1993) Dynamics of the hippocampal ensemble code for space. Science 256:1055-1058.

Young MP, Yamane S (1992) Sparse population coding of faces in the infero-temporal cortex. Science 256:1327-1331.

Young T (1802) II. The Bakerian Lecture. On the theory of light and colors. Philos Trans R Soc Lond 9112-9148.

Zemel RS, Dayan P, Pouget A (1998) Probabilistic interpretation of population codes. Neural Comput 10:403-430.

Zhang K, Ginzburg I, McNaughton BL, Sejnowski TJ (1998) Interpreting neuronal population activity by reconstruction: unified framework with application to hippocampal place cells. J Neurophysiol 79:1017-1044.

Zohary E (1992) Population coding of visual stimuli by cortical neurons tuned to more than one dimension. Biol Cybern 66:265-272

Zoubir AM, Boashash B (1998) The bootstrap and its application in signal processing. IEEE Conference on Communication Systems and Digital Signal Processing, CSDSP, Sheffield, UK, pp 56-76. 